\title{
Detailed Properties of Populous Clusters in the Large Magellanic Cloud
}

\author{
A. J. Grocholski ${ }^{1}$, A. Sarajedini ${ }^{1}$, A. A. Cole $^{2}$, D. Geisler ${ }^{3}$, \\ K. A. G. Olsen ${ }^{4}$, G. P. Tiede ${ }^{5}$, V. V. Smith ${ }^{6}$ and C. L. Mancone ${ }^{1}$ \\ ${ }^{1}$ Deptartment of Astronomy, University of Florida, P.O. Box 112055, Gainesville, FL 32611; \\ email: aaron,ata,cmancone@astro.ufl.edu \\ ${ }^{2}$ Department of Astronomy, University of Minnesota, 116 Church St., S.E., Minneapolis, MN \\ 55455; email: cole@astro.umn.edu \\ ${ }^{3}$ Departamento de Fisica, Universidad de Concepción, Casilla 160-C, Concepción, Chile; \\ email: dgeisler@astro-udec.cl \\ ${ }^{4}$ Cerro Tololo Inter-American Observatory, National Optical Astronomy Observatory, Casilla \\ 603, La Serena, Chile; email: kolsen@noao.edu \\ ${ }^{5}$ Department of Physics and Astronomy, Bowling Green State Univeristy, Bowling Green, $\mathrm{OH}$, \\ 43403; email: gptiede@bgnet.bgsu.edu \\ ${ }^{6}$ Gemini Project, National Optical Astronomy Observatory, Tucson, AZ 85719; \\ email: vsmith@noao.edu
}

\begin{abstract}
We present results from a series of projects aimed at better understanding the ages, velocities, metallicities, and distances of populous clusters in the LMC. Using FORS2 on the VLT, we obtained near-infrared spectra for more than 200 stars in 28 LMC clusters, which span a large range of ages and metallicities. The strong absorption lines of the Ca II triplet are used to calculate cluster velocities and abundances. We determine cluster ages through main sequence fitting of theoretical isochrones to deep optical photometry using a combination of published photometry, VLT FORS2 images and archival HST WFPC2 images. As shown by Grocholski \& Sarajedini, a cluster's age and $[\mathrm{Fe} / \mathrm{H}]$ can be used to predict the $K$-band luminosity of core helium burning red clump (RC) stars in that cluster. Using ISPI on the CTIO $4 \mathrm{~m}$ telescope, we imaged 17 LMC clusters in the $J$ - and $K$-bands, with the resulting photometry reaching $\sim 0.5$ mag below the RC. We combine the measured apparent $\mathrm{RC}$ magnitudes with the predicted absolute values to determine absolute distances to each of these clusters and thereby probe the structure of the LMC as traced by its intermediate-age clusters.
\end{abstract}

Keywords. galaxies: star clusters, Magellanic Clouds, stars: distances, stars: abundances

\section{Introduction}

Due to the proximity of the Milky Way's (MW) satellite galaxies, we are able to resolve and determine physical properties of individual stars, something that is currently not possible with high redshift systems. The Large Magellanic Cloud (LMC), as it is both nearby and located well away from the Galactic plane, provides an excellent opportunity to study the structure and formation history of a satellite galaxy using resolved stars.

Although it is often considered to lie at a single distance from us, the LMC is well known to have a relatively complicated structure. Field star studies have shown that the disk of the LMC is inclined $\sim 35^{\circ}$ with respect to the plane of the sky (e.g. van der Marel \& Cioni 2001). In addition, the disk is thick, flared, and possibly warped (e.g. Alves \& Nelson 2000, Olsen \& Salyk 2002), likely a result of interactions with the Small Magellanic Cloud (SMC) and MW. Only recently, however, has the distribution of the LMC cluster system been explored; using main sequence turnoff (MSTO) fitting 
distances for a sample of 15 intermediate-age clusters, Kerber et al. (2006) showed that, like the LMC's field stars, their clusters have a planar geometry.

In an effort to further explore the geometry of the LMC's cluster system, we calculate absolute cluster distances to a sample of intermediate-age clusters using the $K$-band luminosity of the core helium burning red clump (RC) stars as a standard candle (see Grocholski \& Sarajedini 2002; GS02). These distances not only allow us to determine the spatial distribution of our target clusters, but we are also able to calculate the absolute distance to the center of the LMC. In $\S 2$ we discuss the data necessary to properly employ the $\mathrm{RC}$ as a standard candle and in $\S 3$ we summarize the primary results of our work.

\section{The Data}

As discussed in GS02, both age and $[\mathrm{Fe} / \mathrm{H}]$ have an effect on the $K$-band luminosity of the $\mathrm{RC}\left(M_{K}^{R C}\right)$ and, thus, must be taken into account when using the $\mathrm{RC}$ as a distance indicator. For clusters in the LMC, the work of Olszewski et al. (1991; OSSH) represents the only previous large scale spectroscopic $[\mathrm{Fe} / \mathrm{H}]$ determinations. However, due to limitations of technology at the time, they were only able to observe an average of 2 stars per target cluster. To improve upon their work, we used the FORS2 multi-object spectrograph on the VLT $8.2 \mathrm{~m}$ telescope to obtain moderate-resolution spectra of more than 200 red giant branch stars in 28 LMC clusters. The absorption lines of the calcium II triplet (CaT) were then used to calculate cluster velocities and abundances with small random errors ( $\sigma \sim 1.6 \mathrm{~km} \mathrm{~s}^{-1}$ and $\sigma \sim 0.04$ dex, respectively; Grocholski et al. 2006).

To calculate cluster ages, we have compiled deep optical photometry for a sample of LMC clusters using a combination of published photometry, VLT FORS2 images and archival HST WFPC2 images. These data reach well below each cluster's MSTO and, combined with our spectroscopic abundances, allow us to calculate accurate main sequence fitting ages using theoretical isochrones that include treatment for core overshoot (Grocholski et al. 2007, in prep).

Using the Infrared Side Port Imager on the CTIO $4 \mathrm{~m}$ telescope, we obtained nearinfrared images for 17 intermediate-age LMC clusters, with the resulting photometry reaching $K \sim 18.5$, or about $0.5 \mathrm{mag}$ below the RC (Grocholski et al. 2007). Similar to the approach of GS02, we use our derived cluster ages and metallicities along with an interpolation over the RC models of Girardi \& Salaris (2001) to predict $M_{K}^{R C}$ for each cluster. We then combine $M_{K}^{R C}$, the apparent $K$-band $\mathrm{RC}$ magnitudes measured from our photometry, and cluster reddenings to determine absolute cluster distances.

\section{Results}

The results of our study of the ages, abundances, velocities, and distances of populous clusters in the LMC are now summarized, and we note that points 1-3 are discussed in more detail in Grocholski et al. (2006), point 4 can be found in Grocholski et al. (2007, in prep) and 5-7 are presented in Grocholski et al. (2007).

1. Our derived cluster radial velocities, including six clusters with no previously reported velocities, are in excellent agreement with the work of Schommer et al. (1992), which shows that the LMC cluster system has disk-like rotation with no clusters exhibiting obvious halo kinematics.

2. Similar to previous studies, we find no evidence for a metallicity gradient in the LMC cluster system. This is in stark contrast to stellar populations in both the MW and M33, which have abundances that decrease with increasing galactocentric radius. 


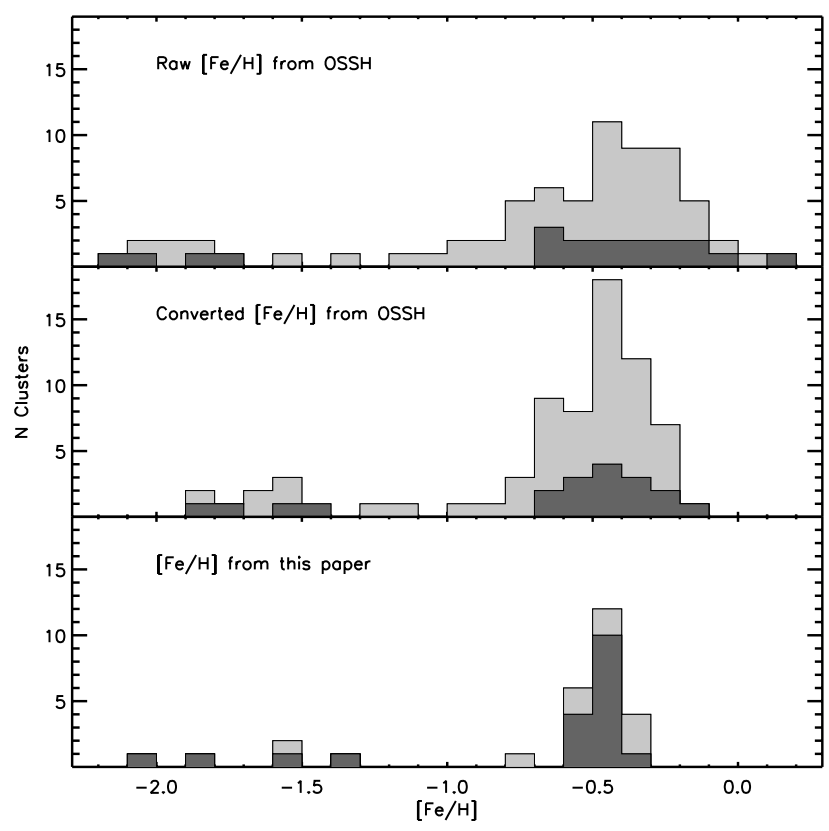

Figure 1. We plot the metallicity distribution of LMC clusters as determined by OSSH and this paper. Published values from OSSH are given in the top panel while the middle panel shows their values converted onto our metallicity scale using Eq. 5 in Grocholski et al. (2006); in the bottom panel we plot our results. In all three panels, the dark shaded region shows the distribution for the 20 clusters in common between OSSH and this paper, while the light shaded region shows the entire cluster sample from each study. Our results indicate that the LMC's intermediate age cluster metallicity distribution is much tighter than suggested by the results of OSSH.

3. The intermediate-age clusters in our sample show a very tight metallicity distribution $([\mathrm{Fe} / \mathrm{H}]=-0.48 \pm 0.09)$, with no tail of clusters approaching solar metallicity. This is in contrast to the work of OSSH, who find a similar mean abundance value, but with a much larger spread in metallicity, as shown in Fig. 1.

4. Cluster ages range from only $\sim 1-3 \mathrm{Gyr}$ for the intermediate-age clusters, and we confirm that ESO 121-SC03, the only cluster in the LMC known to have an age between $\sim 3-13$ Gyr, formed approximately 9 Gyr ago.

5. Absolute cluster distances calculated using the RC method of GS02 allow us to explore the spatial distribution of the LMC cluster system. Previous work has shown that the LMC's field populations reside in a thick, inclined disk; our results, shown in Fig. 2, illustrate that the intermediate age clusters are distributed in the same manner.

6. Using previously published RR Lyrae photometry for seven old LMC globular clusters (e.g., Walker 1993), we calculate their distances and find that, like the intermediateage clusters, the globular cluster distribution is also consistent with the inclined, thick disk geometry of the LMC (see Fig. 2).

7. Taking into account the inclined geometry of the LMC, we use the absolute distances of the intermediate-age clusters to calculate a mean distance to the center of the LMC, which we find to be $(m-M)_{0}=18.40 \pm 0.04_{\text {random }} \pm 0.08_{\text {systematic }}$ or $D_{0}=47.9 \pm 0.9 \pm$ $1.8 \mathrm{kpc}$. Our distance is in excellent agreement with the recent work of Macri et al. (2006) who found $(m-M)_{0}=18.41 \pm 0.1 \pm 0.13$ through a comparison of Cepheid variables in the LMC with those in the maser-host galaxy NGC 4258. This distance, however, is 


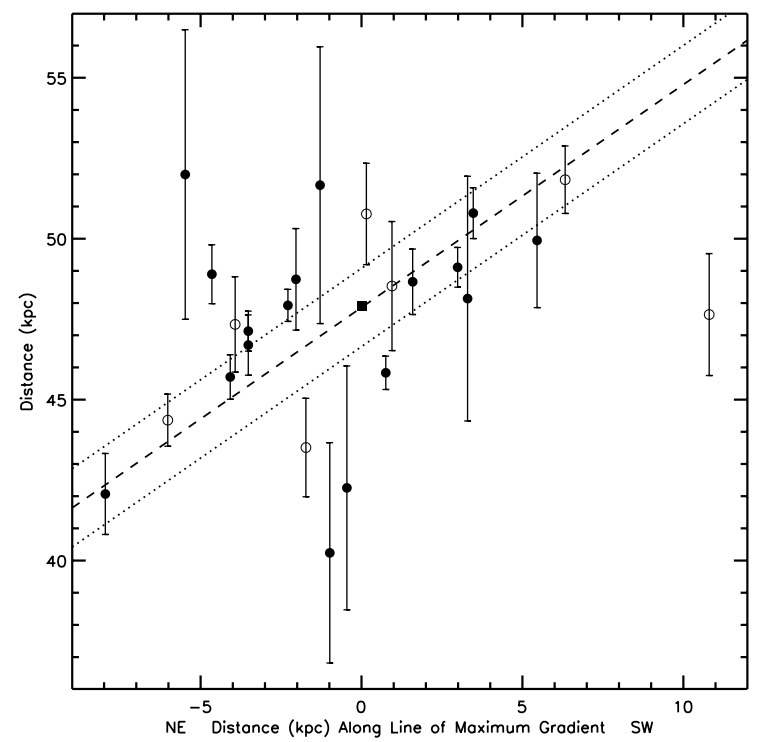

Figure 2. Cluster distances as a function of their position along the line of maximum gradient. Open circles mark the old globular clusters from Walker while the filled circles represent the populous clusters in our study. The dashed line marks the LMC's disk with $i=34.7^{\circ}$ and $D_{0}=47.9 \mathrm{kpc}($ at $x=0$ ), and the dotted lines represent a disk thickness of $\pm 1 \mathrm{kpc}$; the filled square denotes the center of the LMC. This plot illustrates that both the old and intermediate age clusters are distributed along the disk of the LMC.

$\sim 0.1 \mathrm{mag}$ shorter than the commonly accepted distance of $18.5 \pm 0.1 \mathrm{mag}$, which was adopted by the HST Key Project to calculate $H_{0}$ (see Freedman et al. 2001).

\section{Acknowledgements}

This research was supported by NSF CAREER grant AST 0094048 to A.S.

\section{References}

Alves, D.R. \& Nelson, C.A. 2000, ApJ, 542, 789

Freedman, W.L., et al. 2001, ApJ, 553, 47

Grocholski, A.J. \& Sarajedini, A. 2002, AJ, 123, 1603 (GS02)

Grocholski, A.J., Cole, A.A., Sarajedini, A., Geisler, D., \& Smith, V.V. 2006, AJ, 132, 1630

Grocholski, A.J., Sarajedini, A., Olsen, K.A.G., Tiede, G.P., \& Mancone, C.L. 2007, AJ, subm. Kerber, L.O., Santiago, B.X., \& Brocato, E. 2007, A\&\&A, 462, 139

Macri, L.M., Stanek, K.Z., Bersier, D., Greenhill, L., \& Reid, M. 2006, ApJ, 652, 1133

Olsen, K.A.G. \& Salyk, C. 2002, AJ, 124, 2045

Olszewski, E.W., Schommer, R.A., Suntzeff, N.B., \& Harris, H.C. 1991, AJ, 101, 515 (OSSH)

Schommer, R.A., Olszewski, E.W., Suntzeff, N.B., \& Harris, H.C. 1992, AJ, 103, 447

van der Marel, R.P. \& Cioni,M.-R. L. 2001, AJ, 122, 1807

Walker, A.R. 1993, AJ, 105, 527

\section{Discussion}

FERGUSON: It is interesting that you see no evidence for a halo population of clusters yet there are claims for a field halo from RRLyraes. Could you comment on that?.

Grocholski: I am not familiar with the details of the RRLyrae studies but believe the sample size are not very large and are confined to central parts of the galaxy. 